\title{
Oleanolic acid induces protective autophagy in cancer cells through the JNK and mTOR pathways
}

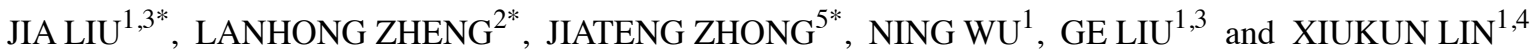 \\ ${ }^{1}$ Institutes of Oceanology, Chinese Academy of Sciences, Qingdao, Shandong 266071; ${ }^{2}$ Yellow Sea Fisheries Research \\ Institute, Chinese Academy of Fishery Sciences, Qingdao, Shandong 266071; ${ }^{3}$ Graduate School, University of Chinese \\ Academy of Sciences, Beijing 100049; ${ }^{4}$ Department of Pharmacology, Capital Medical University, Beijing 100069; \\ ${ }^{5}$ Department of Pathophysiology, Norman Bethune College of Medicine, Jilin University, Changchun, Jilin 130021, P.R. China
}

Received February 28, 2014; Accepted April 16, 2014

DOI: $10.3892 /$ or.2014.3239

\begin{abstract}
Autophagy is a biological process that eliminates damaged or excessive proteins and is utilized by various types of cells to maintain cellular homeostasis. Autophagy also occurs in cancer cells and exerts anti-survival or pro-survival effects depending on stimuli, nutrient and context. Oleanolic acid (OA), a widely spread natural compound, induces apoptosis in a range of cancer cells. However, some tumor cell lines are resistant to the pro-apoptotic effect of OA, and the mechanism remains unknown. In the present study, we found that OA induced autophagic event in cancer cells in a dose- and timedependent manner, evidenced by an increased ratio between LC3-II and LC3-I and frequent granulation of LC3 proteins in OA-stimulated tumor cell lines. Inhibition of autophagy potentiated the pro-apoptotic activity of OA on cancer cells. Furthermore, the JNK and mTOR signaling pathways were found to be affected by OA treatment. Interfering with JNK and mTOR abolished OA-induced autophagy and sensitized cancer cells to apoptosis. Collectively, we showed that OA was able to initiate protective autophagy, which compromised the antitumor activity of OA on cancer cells. Blocking autophagy may be a promising strategy to enhance the tumor suppressor activity of OA.
\end{abstract}

\section{Introduction}

Autophagy is a self-eating mechanism by which cells degrade and renew cellular molecules and organelles. It can be classified

Correspondence to: Dr Lanhong Zheng, Yellow Sea Fisheries Research Institute, Chinese Academy of Fishery Sciences, 7 Nanhai Road, Qingdao, Shandong 266071, P.R. China

E-mail: zhenglanhong@126.com

Dr Xiukun Lin, Department of Pharmacology, Capital Medical University, Beijing 100069, P.R. China

E-mail: linxiukun@yahoo.com

\section{${ }^{*}$ Contributed equally}

Key words: oleanolic acid, autophagy, cancer, mammalian target of rapamycin, $\mathrm{c}$-jun $\mathrm{N}$-terminal kinase as macroautophagy, microautophagy and chaperone-mediated autophagy. Macroautophagy (referred to as autophagy) involves the formation of double membraned vesicles (autophagosomes) containing materials to be degraded, such as unnecessary proteins and damaged or aged organelles. Autophagosome then fuse with lysosomes, break down the cargos and recycle the useful molecules. Autophagy at a basal level is essential for cellular clearance of unwanted materials. Dysregulated autophagy leads to dysfunction and even death of cells, due to the cytotoxicity of the accumulated materials. The role of autophagy in cancer biology depends on the context. In normal cells, autophagy contributes to the prevention of carcinogenesis by eliminating the molecules that may induce or promote cancer formation. On the other hand, established cancer cells utilize autophagy to survive some adverse conditions, such as hypoxia, low nutrition and oxidative stress.

Oleanolic acid [(3ß)-3-hydroxyolean-12-en-28-oic acid], a natural pentacyclic triterpenoid that exists widely in various type of plants, is believed to be one of the most important compounds with bioactivity in vegetables and medicinal herbs (1). Its bioactivity includes anti-inflammation, antivirus, hepatoprotection and antitumor (1). OA displayed no significant cytotoxicity to normal cells (1). Owing to these characteristics, researchers are making efforts to improve its activity by developing its derivatives $(2,3)$. Considering that cancer is a severe risk to human health, the antitumor activity of OA has attracted increasingly more attention from researchers. As the major antitumor mechanism, OA induces apoptotic death in a wide range of cancer cells $(4,5)$. OA stimulation leads to an increase in the ratio between the proapoptotic protein Bax and the antiapoptotic protein $\mathrm{Bcl}-2$, in the mitochondrial membrane, which promotes Bax to oligomerize. The permeability of mitochondrial membrane was consequently elevated, which facilitates the release of cytochrome $c$ into cytoplasm. The caspase family proteases were, therefore, cleaved and activated.

However, some cells were found to be resistant to OA-induced apoptosis. The mechanisms underlying the resistance remain unknown. In the present study, we found that OA was able to induce autophagy in cancer cells. Autophagy impaired the pro-apoptotic effect of OA on cancer cells. Blocking autophagy restored the sensitivity of cancer cells to OA-induced apoptotic death. 


\section{Materials and methods}

Cell lines. A549, MCF-7, U2OS, BXPC3, PANC-1 and PC-3 cells were purchased from the American Type Culture Collection (ATCC; Manassas, VA, USA) and cultured in the recommended media supplemented with $10 \%$ fetal bovine serum (FBS; Invitrogen), $4 \mathrm{mM}$ glutamine, $100 \mathrm{U} / \mathrm{ml}$ penicillin. All the cells were cultured in a humidified $5 \% \mathrm{CO}_{2}$ atmosphere at $37^{\circ} \mathrm{C}$.

Chemical reagents. Oleanolic acid (OA; O5504) and autophagy inhibitor 3-methyladenine (3-MA; M9281) were purchased from Sigma-Aldrich. Atg7-targeting siRNAs (siATG7) (\#6604) and control siRNA (siControl) (\#6568) were purchased from Cell Signaling Technology (Beverly, MA, USA). Inhibitors that selectively blocked JNK (SP600125, \#8177) were purchased from Cell Signaling Technology. mTOR activator MHY1485 (2 $\mu \mathrm{M})$, Millipore, \#5.00554.0001.

$p$ GFP-LC3 transfection. Cancer cells were seeded on the top of coverslips in a 24-well plate. The cells were transfected with the plasmid, pEGFP-LC3, using Lipofectamine 2000 (Invitrogen) following the manufacturer's instruction. Forty-eight hours after transfection, cells were treated with $100 \mu \mathrm{g} / \mathrm{ml}$ of OA for $12 \mathrm{~h}$. The cells were then fixed with $4 \%$ polyoxymethylene and observed under an Olympus FluoView ${ }^{\mathrm{TM}}$ FV1000 confocal microscope (Olympus, Hamburg, Germany). Cells with five GFP-LC3 puncta or more were counted per 100 random cells in each group.

Immunoblotting assay. Proteins of human tissues or cultured cells were harvested with M-PER Mammalian Protein Extraction reagent (Thermo Fisher Scientific, Rockford, IL, USA), separated using polyacrylamide gel electrophoresis and transferred onto $0.45-\mu \mathrm{m}$ nitrocellulose membranes. The membranes were blocked with 5\% fat-free dry milk in phosphate-buffered saline (PBS) and incubated with primary antibodies at $4^{\circ} \mathrm{C}$. Overnight, the membrane was incubated with corresponding secondary antibodies and visualized with SuperSignal West Dura Extended Duration Substrate (Thermo Fisher Scientific).

The involved primary antibodies in the present study included: LC3B, \#3868; Beclin-1, \#3495; cleaved PARP, CST, \#5625; cleaved caspase-3 CST, \#9664; cleaved caspase-9, CST, \#9501; cytochrome $c$, GST, \#11940; phospho-JNK (Thr183/ Tyr185), \#4668; JNK, \#9258; phospho-mTOR (Ser2448), \#5536; mTOR, \#2983. The intensity of blots in each figure was determined with ImageJ software.

Apoptotic rate determination by flow cytometry. The rates of apoptotic cells were evaluated by flow cytometric analysis. Briefly, cells were fixed in cold $70 \%$ ethanol for $1 \mathrm{~h}$, and were then incubated with $10 \mathrm{mg} / \mathrm{ml}$ RNase A at $37^{\circ} \mathrm{C}$ for $1 \mathrm{~h}$. The cells were stained with propidium iodide (PI; $200 \mathrm{mg} / \mathrm{ml}$ ), and immediately subjected to flow cytometric analysis (BD FACSAria II; BD Biosciences). Cells $\left(1 \times 10^{5}\right)$ were counted for each sample.

Cell viability assay. Cancer cells $\left(1 \times 10^{4}\right)$ were seeded in 96-well plates. Fifty milliliters of 3-(4, 5-dimethylthiazol- 2-yl)-2,5-diphenyltetrazolium bromide (MTT) $(1 \mathrm{mg} / \mathrm{ml})$ was used at the indicated time-points. After $4 \mathrm{~h}$, MTT was removed and $150 \mathrm{ml}$ of dimethyl sulfoxide (DMSO) was then added. The absorbance at $570 \mathrm{~nm}$ was evaluated on a model 550 microplate reader (Bio-Rad Laboratories, Hercules, CA, USA) with a reference wavelength of $655 \mathrm{~nm}$.

Statistical analysis. The experiments, with the exception of the immunoblot assays, were performed at least three times. All values are reported as means $\pm \mathrm{SD}$, and compared at a given time-point by unpaired, two-tailed t-test. Differences were considered statistically significant when ${ }^{*} \mathrm{P}<0.05$ and ${ }^{* *} \mathrm{P}<0.01$.

\section{Results}

OA induces autophagy in a wide range of cancer cells in a dose- and time-dependent manner. Autophagy has been reported to be initiated in neoplastic cells by natural and synthetic compounds (6,7). First, we investigated if OA induced autophagy in cancer cells by immunoblot analysis of light chain 3 (LC3) isoforms. OA was found to increase the expression level of LC3-II in various cancer cell lines. This effect occurred in a dose- and time-dependent manner (Fig. 1A and 1B). Autophagic event was further confirmed by observing the fluorescence in the cancer cells transfected with pGFP-LC3, a plasmid expressing a fusion protein that tracks the location of LC3. The GFP-LC3 puncta were frequently seen in the cancer cells treated with OA, whereas the control cells had a relatively homogeneous LC3 expression pattern (Fig. 1C).

Blocking autophagy potentiates the apoptotic death of cancer cells treated with $O A$. Considering the role of autophagy in apoptotic pathways in cancer cells, we aimed to determine the effect of autophagic events in OA-induced apoptosis in cancer cells. A small molecule inhibitor of autophagy, 3-MA, was used to abolish the occurrence of autophagy induced by OA. Cell viability was shown to be further reduced when OA and 3-MA was added to the media (Fig. 2A). Immunoblot assays revealed that 3-MA suppression of autophagy was able to enhance the cleavage and activation and caspase family proteases as well as PARP, which are all the biomarkers of apoptosis induction (Fig. 2B). Consistently, higher sub- $\mathrm{G}_{0} / \mathrm{G}_{1}$ population was detected in cancer cells co-treated with OA and 3-MA, in comparison with the cells treated with OA alone (Fig. 2C). A siRNA silencing Atg7 expression (siATG7) was also employed to suppress autophagic event in OA-incubated cancer cells. Similar to the results obtained from 3-MA-based assays, siATG7 also synergistically decreased the survival of cancer cells, potentiated the activation of apoptotic pathways, and heightened the percentage of sub- $\mathrm{G}_{0} / \mathrm{G}_{1}$ population with OA, in cancer cells (Fig. 2D-F). These data supported the hypothesis that autophagy induced by OA exerted a suppressive effect on apoptotic pathways in cancer cells.

$O A$ induces JNK activation and mTOR suppression in cancer cells. To study the mechanisms by which OA induced autophagy, we focused on c-Jun N-terminal kinase 1 (JNK) and MTOR signaling cascades. JNK is closely associated with the initiation of autophagic events in a variety of cell types. Activated JNK-1 phosphorylates N-terminal region of Bcl-2 


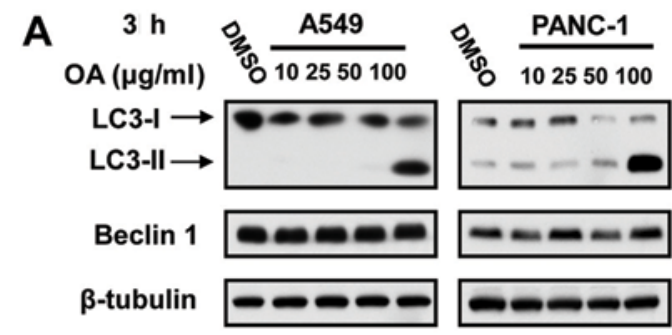

C

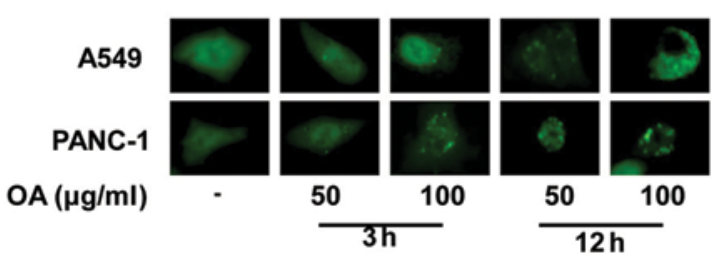

B

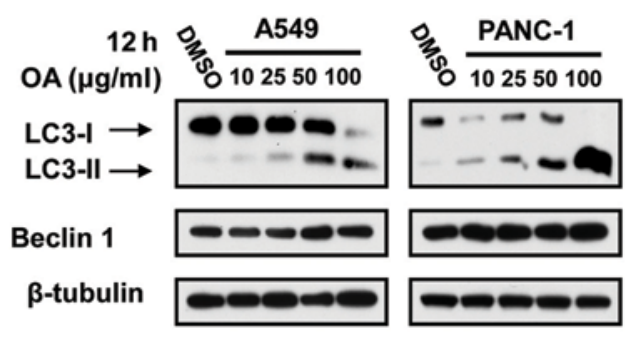

D

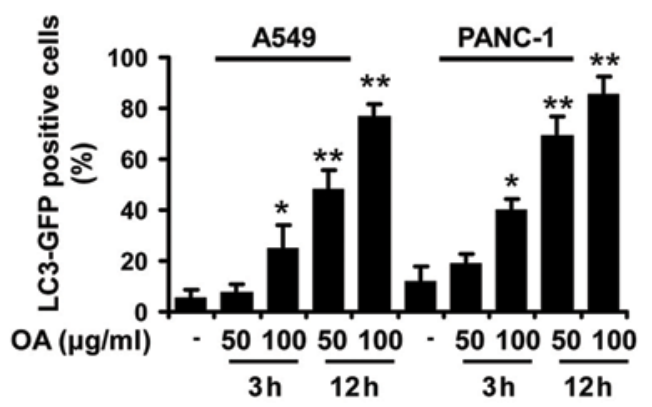

Figure 1. OA induces autophagy in cancer cells. A549 and PANC-1 cells were treated with OA $(10,25,50 \mathrm{or} 100 \mu \mathrm{g} / \mathrm{ml})$. (A) Three or (B) $12 \mathrm{~h}$ later, the expression of LC3-I, LC3-II and Beclin 1 was detected by immunoblot assays. A549 and PANC-1 cells were transfected with a plasmid expressing LC-3-GFP. (C and D) Three or $12 \mathrm{~h}$ later, the cells containing GFP functates were counted by immunofluorescent microscopy.

A

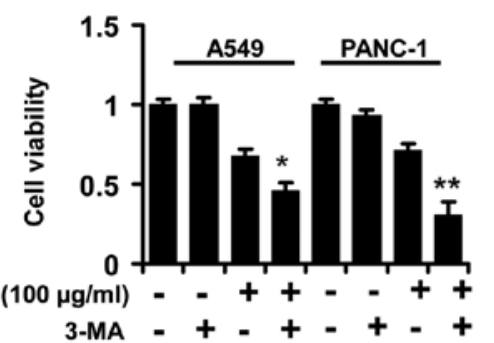

B

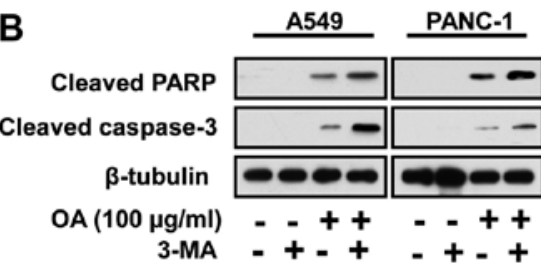

C

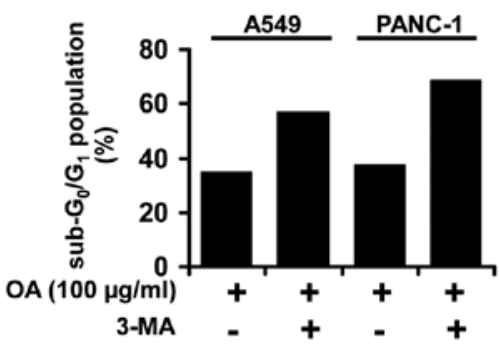

D

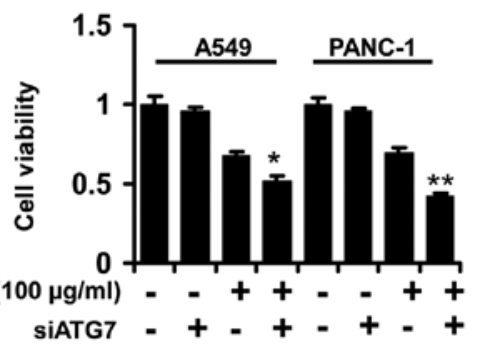

E

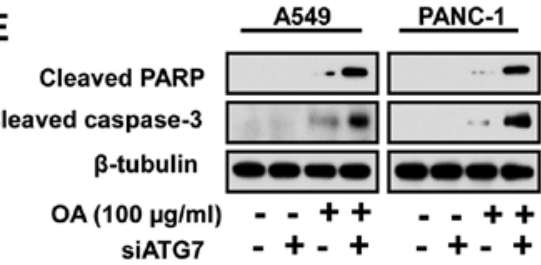

$\mathbf{F}$

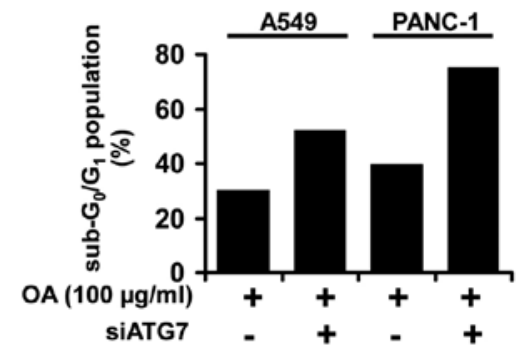

Figure 2. The suppression of autophagy enhances the pro-apoptotic effect of OA on cancer cells. (A) A549 and PANC-1 cells were treated without or with OA $(100 \mu \mathrm{g} / \mathrm{ml})$ in the presence and absence of 3-MA. Twelve hours later, cell viability was evaluated by MTT assays. (B) The expression of cleaved caspase-3 and PARP was detected by immunoblot analysis. (C) The percentages of sub- $\mathrm{G}_{0} / \mathrm{G}_{1}$ population were quantified by flow cytometry. (D) A549 and PANC-1 cells were treated without or with OA $(100 \mu \mathrm{g} / \mathrm{ml})$ in the presence and absence of siATG7. Twelve hours later, cell viability was evaluated by MTT assays. (E) The expression of cleaved caspase- 3 and PARP was detected by immunoblot analysis. (F) The percentages of sub- $\mathrm{G}_{0} / \mathrm{G}_{1}$ population were quantified by flow cytometry.

and triggers its dissociation from Beclin 1, the most potent inducer of autophagic pathway (8). The released Beclin 1 subsequently induced the assembly of autophagosomes. OA was found to induce the JNK activation in a range of cancer cells, in a time-dependent manner (Fig. 3). mTOR has been well documented as the major suppressor of cellular autophagy. 

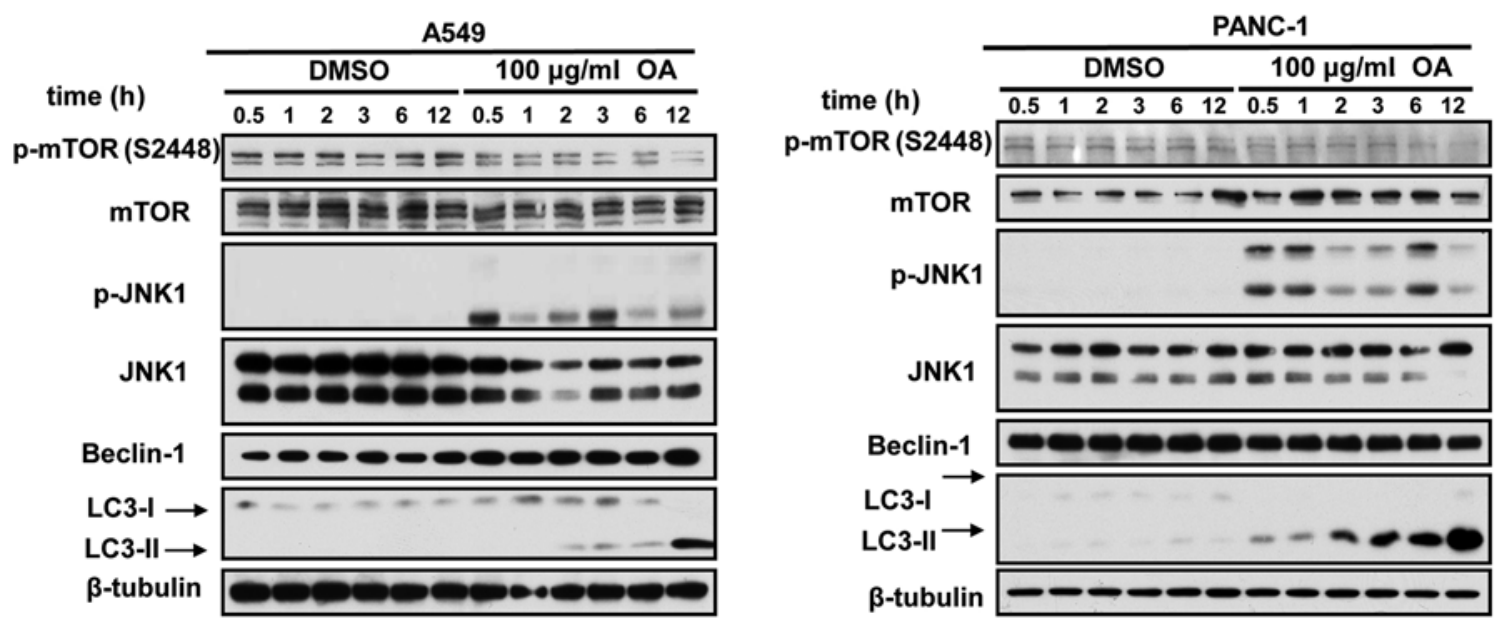

Figure 3. OA suppresses and triggers the activation of mTOR and JNK pathways, respectively, in cancer cells. The expression of p-mTOR, mTOR, p-JNK and JNK was detected by immunoblot assays, as well as LC3-I, LC3-II and Beclin 1, when A549 and PANC-1 was treated with OA (100 $\mu \mathrm{g} / \mathrm{ml})$ at the indicated time-points.

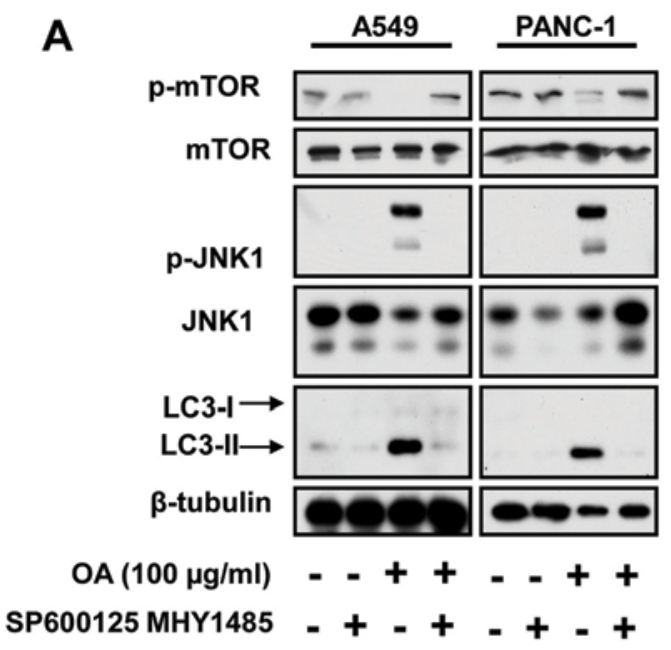

B
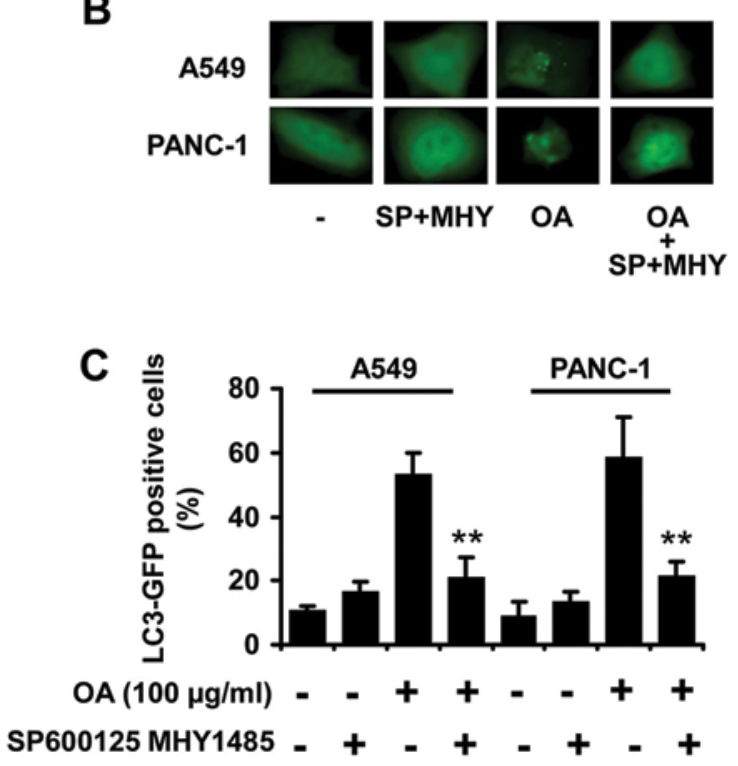

Figure 4. OA induction of autophagy requires the suppression of the mTOR and activation of the JNK pathways. (A) A549 and PANC-1 cells were treated without or with OA $(100 \mu \mathrm{g} / \mathrm{ml})$ in the presence and absence of SP600125 and MHY1485. Twelve hours later, the expression of p-mTOR, mTOR, p-JNK, JNK, LC3-I, LC3-II and Beclin 1 was detected by immunoblot assays. (B and C) A549 and PANC-1 cells were transfected with a plasmid expressing LC-3-GFP. Twelve hours later, the cells containing GFP puncta were counted by immunofluorescent microscopy.

Immunoblot assays also indicated that mTOR phosphorylation was diminished in cancer cells under the treatment of OA (Fig. 3).

The alteration in JNK and $m$ TOR activitymediates $O A$-induced autophagy. Having established that JNK activation and mTOR suppression was caused by OA treatment in cancer cells, we subsequently investigated whether the changes in their activity accounted for the occurrence of autophagic events. SP600125 and MHY1485 were used to inhibit JNK activation and reactivate mTOR, respectively. OA-related autophagy was abrogated by these two compounds, indicated by the reduction in LC3-II expression (Fig. 4A), as well as the amount of LC3 puncta (Fig. 4B and C).
JNK suppression and mTOR activation enhance the apoptosis induced by $O A$. To further confirm the requirement of JNK suppression and mTOR activation in the effect of OA on cancer cells, we employed SP600125 and MHY1485 to change the active status of these two pathways. The data revealed that SP600125 and MHY1485 potentiated the antitumor activity of OA on A549 and PANC-1 cells (Fig. 5A). Furthermore, the activation of apoptosis-related proteins was also detected by immunoblot assays. SP600125 and MHY1485 led to increased expression of cleaved caspase-3 and PARP in cancer cells co-treated with OA (Fig. 5B). Also, the percentage of sub- $G_{0} / G_{1}$ was higher in cancer cells when the JNK and mTOR pathways were suppressed and activated, respectively (Fig. 5C). 
A
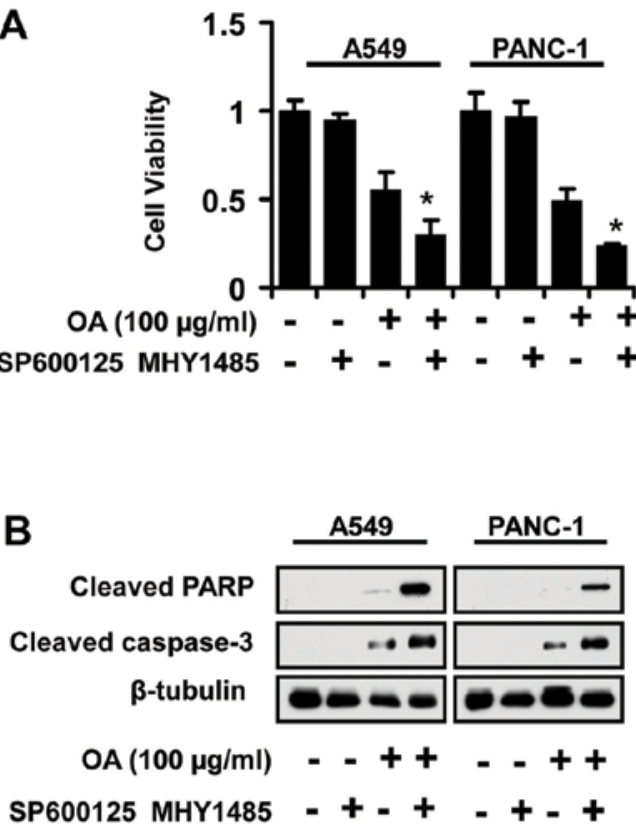

C

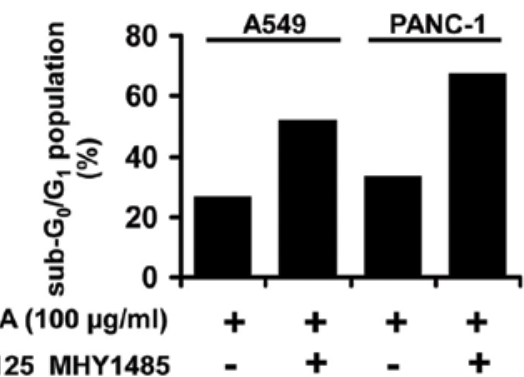

D

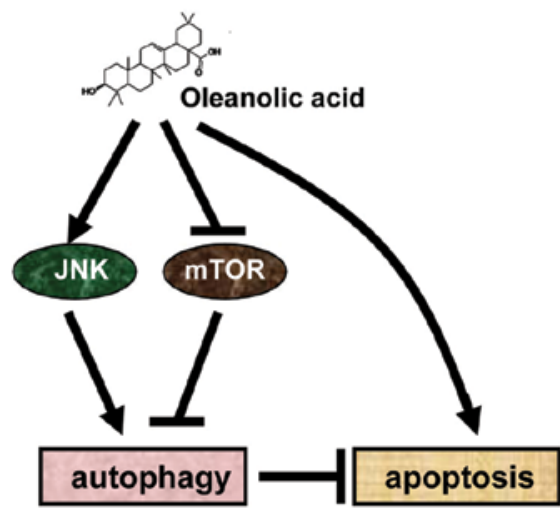

Figure 5. The suppression of the mTOR and activation of the JNK pathways abolished the antitumor activity of OA on cancer cells. (A) A549 and PANC-1 cells were treated without or with OA $(100 \mu \mathrm{g} / \mathrm{ml})$ in the presence and absence of SP600125 and MHY1485. Twelve hours later, cell viability was evaluated by MTT assays. (B) The expression of cleaved caspase-3 and PARP was detected by immunoblot analysis. (C) The percentages of sub-G0/G 1 population were quantified by flow cytometry. (D) Illustration of the mechanism by which OA induced autophagy and its association with apoptosis.

\section{Discussion}

Although OA treatment has been demonstrated to trigger the onset of many cellular processes, such as apoptosis (5), cell cycle arrest (9) and impaired motility (10), it remains unknown if OA can induce autophagic events in cancer cells. Autophagy is a biological process widely employed by a wide range of cells to defend themselves against adverse stimuli, including the treatment of antitumor compounds. In fact, it has been reported that autophagy can be induced by many bioactive natural compounds in cancer cells (6). However, the role of induced autophagy in the antitumor potency of natural compounds appears to vary between different compounds. Curcumin (11), $\alpha$-eleostearic acid (12), evodiamine (13) and resveratrol (14), have been shown to result in cell death by inducing autophagy. In contrast, cancer cells employ autophagy as a protective mechanism against the antitumor activity of isobavachalcone (15) and quercetin (16). In the present study, we experimentally indicated that OA was able to induce autophagy in cancer cells, which can enhance the resistance of cancer cells to apoptosis. To the best of our knowledge, this is the first study to provide evidence that autophagy can be triggered by OA stimulation. Furthermore, OA-induced autophagy may account for the resistance against apoptosis of some types of cancer cells.

The pathways that initiate or maintain the activation of autophagy have been well studied. Among them, JNK and mTOR pathways are the most important (17). It has also been well documented that these two pathways mediated the onset of autophagy in cancer cells under the treatment of some compounds $(11,18)$. Previously, OA or its derivative was demonstrated to activate the JNK pathway both in normal cells (19) and malignant cells (20), as well as the suppression of mTOR pathway (21). The outcome of these alterations was mainly limited to apoptosis and cell cycle arrest. It remains unexplored if other effects result from JNK activation and mTOR suppression by OA. The present study revealed, for the first time, that OA's effect on JNK and mTOR activation lead to autophagy in cancer cells.

In conclusion, OA induced protective autophagy in cancer cells by JNK activation and mTOR inhibition, which enhanced the resistance of cancer cells to apoptotic events. Our findings contribute to a better understanding of OA's action mode. Furthermore, we provided evidence that suppression of autophagy is an effective strategy to enhance antitumor activity of OA.

\section{Acknowledgements}

The present study was supported by the National Innovative Drug Development Projects (2014ZX-09102043-001). The study was also supported in part by the National Natural Science Foundation of China (81302906, 81273550 and 41306157).

\section{References}

1. Pollier J and Goossens A: Oleanolic acid. Phytochemistry 77: 10-15, 2012. 
2. Liby KT, Yore MM and Sporn MB: Triterpenoids and rexinoids as multifunctional agents for the prevention and treatment of cancer. Nat Rev Cancer 7: 357-369, 2007.

3. Petronelli A, Pannitteri G and Testa U: Triterpenoids as new promising anticancer drugs. Anticancer Drugs 20: 880-892, 2009.

4. Chakravarti B, Maurya R, Siddiqui JA, et al: In vitro anti-breast cancer activity of ethanolic extract of Wrightia tomentosa: role of pro-apoptotic effects of oleanolic acid and urosolic acid. J Ethnopharmacol 142: 72-79, 2012.

5. Wei J, Liu M, Liu H, et al: Oleanolic acid arrests cell cycle and induces apoptosis via ROS-mediated mitochondrial depolarization and lysosomal membrane permeabilization in human pancreatic cancer cells. J Appl Toxicol 33: 756-765, 2012.

6. Zhang X, Chen LX, Ouyang L, Cheng Y and Liu B: Plant natural compounds: targeting pathways of autophagy as anti-cancer therapeutic agents. Cell Prolif 45: 466-476, 2012.

7. Livesey KM, Tang D, Zeh HJ and Lotze MT: Autophagy inhibition in combination cancer treatment. Curr Opin Investig Drugs 10: 1269-1279, 2009.

8. Wei Y, Pattingre S, Sinha S, Bassik M and Levine B: JNK1mediated phosphorylation of Bcl-2 regulates starvation-induced autophagy. Mol Cell 30: 678-688, 2008.

9. Wang X, Bai H, Zhang X, et al: Inhibitory effect of oleanolic acid on hepatocellular carcinoma via ERK-p53-mediated cell cycle arrest and mitochondrial-dependent apoptosis. Carcinogenesis 34: 1323-1330, 2013.

10. Guo G, Yao W, Zhang Q and Bo Y: Oleanolic acid suppresses migration and invasion of malignant glioma cells by inactivating MAPK/ERK signaling pathway. PLoS One 8: e72079, 2013.

11. Aoki H, Takada Y, Kondo S, Sawaya R, Aggarwal BB and Kondo Y: Evidence that curcumin suppresses the growth of malignant gliomas in vitro and in vivo through induction of autophagy: role of Akt and extracellular signal-regulated kinase signaling pathways. Mol Pharmacol 72: 29-39, 2007.
12. Eom JM, Seo MJ, Baek JY, et al: Alpha-eleostearic acid induces autophagy-dependent cell death through targeting AKT/mTOR and ERK1/2 signal together with the generation of reactive oxygen species. Biochem Biophys Res Commun 391: 903-908, 2010.

13. Rasul A, Yu B, Zhong L, Khan M, Yang H and Ma T: Cytotoxic effect of evodiamine in SGC-7901 human gastric adenocarcinoma cells via simultaneous induction of apoptosis and autophagy. Oncol Rep 27: 1481-1487, 2012.

14. Filippi-Chiela EC, Villodre ES, Zamin LL and Lenz G: Autophagy interplay with apoptosis and cell cycle regulation in the growth inhibiting effect of resveratrol in glioma cells. PLoS One 6: e20849, 2011.

15. Zhao S, Ma CM, Liu CX, et al: Autophagy inhibition enhances isobavachalcone-induced cell death in multiple myeloma cells. Int J Mol Med 30: 939-944, 2012.

16. Wang K, Liu R, Li J, et al: Quercetin induces protective autophagy in gastric cancer cells: involvement of Akt-mTOR- and hypoxiainduced factor 1 $\alpha$-mediated signaling. Autophagy 7: 966-978, 2011.

17. Mehrpour M, Esclatine A, Beau I and Codogno P: Overview of macroautophagy regulation in mammalian cells. Cell Res 20 : 748-762, 2010.

18. Xavier CP, Lima CF, Pedro DF, Wilson JM, Kristiansen K and Pereira-Wilson C: Ursolic acid induces cell death and modulates autophagy through JNK pathway in apoptosis-resistant colorectal cancer cells. J Nutr Biochem 24: 706-712, 2013.

19. Wang X, Ye XL, Liu R, et al: Antioxidant activities of oleanolic acid in vitro: possible role of Nrf2 and MAP kinases. Chem Biol Interact 184: 328-337, 2010.

20. Zou W, Yue P, Khuri FR and Sun SY: Coupling of endoplasmic reticulum stress to CDDO-Me-induced up-regulation of death receptor 5 via a CHOP-dependent mechanism involving JNK activation. Cancer Res 68: 7484-7492, 2008.

21. Zhou R, Zhang Z, Zhao L, et al: Inhibition of mTOR signaling by oleanolic acid contributes to its anti-tumor activity in osteosarcoma cells. J Orthop Res 29: 846-852, 2011. 